\title{
A Generalized S transform and TT Transform Based on Energy Normalized Window
}

\author{
Jicheng Liu ${ }^{1}$, Jianhong Yao ${ }^{1}$ and Xia Liu ${ }^{1}$ \\ ${ }^{1}$ Northeast Petroleum University, Daqing, Heilongjiang 163318, People' Republic of \\ China \\ ljcdqpi@163.comyjhljc@163.comliuxia2k@163.com
}

\begin{abstract}
A generalized $S$ transform based on energy normalized windows is presented, which avoids the weighting effect of existing $S$ transform influenced by the time-frequency spectral energy. The transform can reflect the spectral energy distribution of different frequency components while maintaining the excellent time-frequency resolution. While TT transform is a new approach to process non-stationary signal based on $S$ transform, which has differential concentration for different frequency components, and lower frequency components can be suppressed through extracting the TT spectrum's diagonal elements. However, this method has a deficiency that part of high frequency interference is retained during processing low frequency signal. Therefore, time-frequency filtering based on the generalizing $S$ transform is used to suppress the high frequency interference and TT transform is adopted to suppress the low frequency contents. Finally, this method is used to process seismic data, the result demonstrates the effective of combining energy normalized $S$ transform and TT transform.
\end{abstract}

Keywords: Time-frequency analysis, S transform, TT transform, Signal filtering

\section{Introduction}

The time-frequency analysis method maps one dimension time or frequency domain signal into two dimension time-frequency plane, then the time-frequency distribution of signal and signal components can be obtained. The commonly used time-frequency analysis methods include STFT (Short Time Fourier Transform), Gabor transform, Wigner-Ville distribution and wavelet transform etc., [1-2]. All of these methods have advantages and drawback respectively. The time-frequency resolution of STFT is stationary because it uses a fixed sliding window to obtain time-localized spectra. So it exhibits poor time resolution at high frequencies, and can't accurately resolve low frequencies whose periods are longer than the duration of the window. Wavelet transform provides a different approach of time-frequency analysis, and it produces a time-scale map called scalogram. As scale represents a frequency band, it is not intuitive to interpret the frequency content of the signal.

The ideal time-frequency transform only provides information about the frequency occurring at a given time instant; it attempts to combine the local information of an instantaneous-frequency spectrum with the global information of the temporal behavior of the signal. So the different time-frequency analysis methods are to obtain time-varying spectrum functions with high resolution and to overcome potential interferences. S transform induced by Stockwell is the extension of wavelet transform whose kernel function is the product of simple harmonic wave and Gaussian function [3]. So, it can conceptually be viewed as a hybrid of short time Fourier analysis and wavelet analysis. The frequency-dependent window function produces higher frequency resolution at lower frequencies, while at higher 
frequencies, sharper time localization can be achieved. Compared with the time-frequency analysis methods such as wavelet transform and STFT etc., S transform has unique characteristics. The time-frequency resolution associates with frequency, which not only has multi-scale concentration, but also has direct relationship with Fourier spectrum. It can maintain absolutely phase of frequency, and its basic transform need not to suffice for admissibility. For these reasons, the $\mathrm{S}$ transform has already been considered in many fields such as geophysics, cardiovascular time series analysis, signal processing for mechanical system, power system engineering, and pattern recognition [4-9].

TT (Time-Time) transform is time-time analysis method proposed by Pinnegar in 2003 based on S transform, which localized signal and is 2D time-time representation of 1D time signal [10]. The validity of TT transform has been proved by using TT filter [11]. Fan X used TT transform to gearbox fault detection and obtained satisfactory results [12-13]. In the applications using the TT, only its diagonal part has been used. Simon proposed the method of computing diagonal elements of TT spectrum, which improves computational efficiency and makes TT transform more practical [14].

TT transform is rooted in $\mathrm{S}$ transform, although several non-Gaussian windows have been used to obtain variant $S$ transforms [15-16], the Gaussian window is the most common to obtain the TT-transform. In this paper, a generalized S transform based on energy normalized window and corresponding TT transform is introduced, some of its properties are described. According to [11], a joint time-frequency filtering is used to process seismic signal combining the energy normalized window $\mathrm{S}$ transform and its TT transform.

\section{S Transform and TT Transform}

The S Transform of a time series $u(t)$ is defined as:

$$
S(\tau, f)=\int_{-\infty}^{+\infty} u(t) w(\tau-t, f) \exp (-i 2 \pi f t) d t
$$

With Gaussian window:

$$
w(\tau-t, f)=\frac{|f|}{\sqrt{2 \pi} k} \exp \left(-f^{2}(\tau-t)^{2} / 2 k^{2}\right)
$$

Where $\mathrm{f}$ is frequency and $\tau$ and $t$ is time variables, $\tau$ is the center time of the Gaussian window and $k$ is a regulating parameter of the width of the window, $k>0$, which also controls the time-frequency resolution. $k$ may be increased for increasing frequency resolution, with a corresponding loss of resolution in time. When $k=1,(1)$ is the standard $\mathrm{S}$ transform.

An important constraint on $\mathrm{w}$ is the normalization condition:

$$
\int_{-\infty}^{+\infty} w(\tau-t, f) d \tau=1
$$

According to the fact that the Fourier transform of a Gaussian is a Gaussian, Stockwell, et al., provides the terms of the Fourier transform of the signal:

$$
S(\tau, f)=\int_{-\infty}^{+\infty} U(\alpha+f) \exp \left(-2 \pi^{2} \alpha^{2} k^{2} / f^{2}\right) \exp (i 2 \pi \alpha \tau) d \alpha
$$

Where $U(\alpha)$ is the Fourier transform of $u(t)$ and computed only once. $U(\alpha)$ is shifted by $f$ before it is multiplied with the Gaussian window.

Due to (1) and (3), $S$ collapses onto $U$, the Fourier transform of $u(t)$, when integrated over all range of $\tau$ : 


$$
U(f)=\int_{-\infty}^{+\infty} S(\tau, f) d \tau
$$

Then, the inverse DFT of (5) gives the inverse S transform:

$$
u(t)=\int_{-\infty}^{+\infty} \int_{-\infty}^{+\infty} S(\tau, f) \exp (i 2 \pi f t) d \tau d f
$$

Computing the inverse Fourier transform of (1),

$$
T T(\tau, t)=\int_{-\infty}^{+\infty} S(\tau, f) \exp (i 2 \pi f t) d f
$$

Formula (7) is called TT transform, where $\tau$ describes the time position of the $\mathrm{S}$ transform window, $t$ is the time axis of the original time series. In the whole range of $\tau$, the result of (7) is local time function for particular $t$, which expresses the amplitude change of signal with time and frequency. For particular $t$, the columns of the TT-transform are the Fourier transform pairs of the corresponding columns of the S-transform. Every column of the TTtransform is called a TT-series and it is a time-local time series. The different frequency is corresponding to different windows in TT transform, which is distinguished from windowed short Fourier transform. The high frequency components focus on the center of the Gaussian window more than low frequency components. As the scaling characteristic of TT transform, the amplitude of high frequency contents is larger than that of low frequency contents near $|\tau-t|$, and with increasing of $|\tau-t|$; high frequency components attenuate more quickly than low frequency. The original signal can be gained by summing TT series for all $\tau$, so TT transform is invertible and its invertible transform:

$$
u(t)=\int_{-\infty}^{+\infty} T T(\tau, t) d \tau
$$

Figure 1 (a) is test signal and (b) is its $\mathrm{S}$ transform. Fig.1(c) is the TT transform and each column is a localized time series which is called TT series. According to (8), (d) is the reconstructed signal produced by summing all the columns of (c). (e) and (f) are the TT series corresponding to different $\tau$. When $\tau=49$, the amplitude of high frequency component is bigger than that of low frequency. But it is contrary in test signal. $\tau=127$ Is the boundary point of low frequency and mid-frequency? The amplitude of mid-frequency is higher than that of low frequency in $\tau=t$, but the amplitude is same in test signal. With the increasing of $|\tau-t|$ (for example $\tau=49$ ), high frequency components attenuate rapidly compared to low frequency components. Mid-frequency components attenuate rapidly compared to low frequency components at $\tau=127$. So, high frequency contents concentrate at $\tau=t$ still more than low frequency contents, which are called signal localization with different frequency.

The localization of signal can limit the band of TT series within one to two wavelength at $\tau=t$, as shown in $\tau=89, \tau=159$ and $\tau=199$. The extremums and zero points of TT series are closer to $\tau=t$ than that of original series that is time shift characteristic of extremums and zero points, which can't be observed in windowed time series. The amplitude of TT series is more and more small farther away from $\tau=t$, and the amplitude is very small to a certain degree distance, which makes the wavelength is very long and the extremums and zero points of TT series is far away from $\tau=t$ compared to that of test series. If the window function is monotonous or constant in two directions such as Hanning Window, the phenomenon doesn't appear for windowed time series. As shown in Fig.1(c), three frequency contents of the signal have analogous shape, but their shape varies with frequency in 
continuous domain and the change can't be observed in STFT. Three frequency contents of signal in Fig.1(c) have same width in $\tau$ coordinate axis and different width in t coordinate axis. If the different frequency contents of signal overlap in time-amplitude chart, the interference signal will emerge in spectrogram, $\mathrm{S}$ transform, TT transform and TT series, which maybe wrong polarity of extremums.

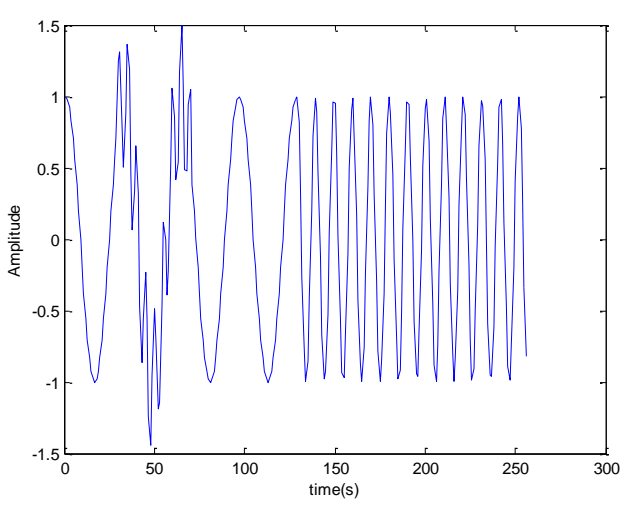

(a)

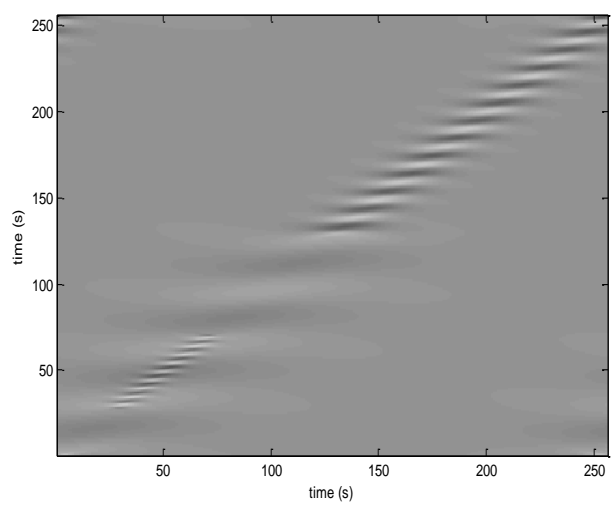

(c)
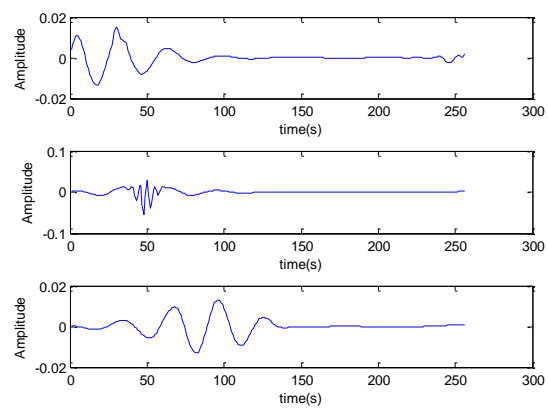

(e)

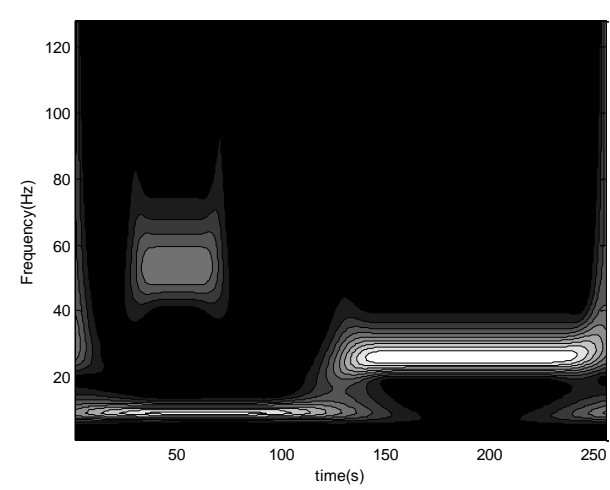

(b)

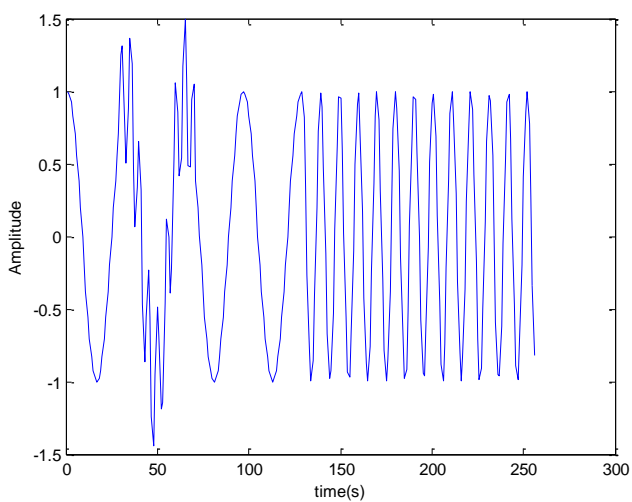

(d)
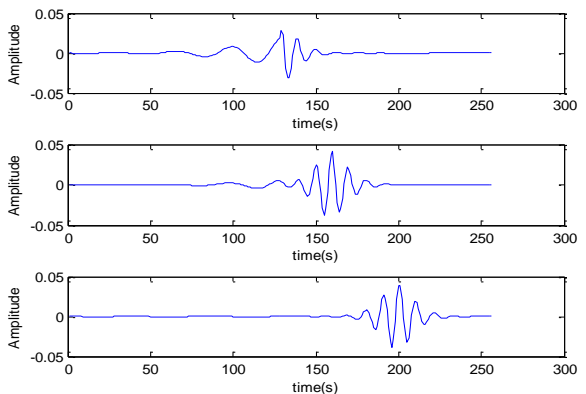

(f)

Figure 1. (a) Testing Signal. (b) S Transform of (a). (c) TT Transform of (a). (d) Reconstructed Time Series Produced by Summing All the Columns of Subplot (c). (e) TT-Series of Subplot (c) at $\mathrm{T}=19,49,89$ (from Top to Bottom). (f)TT-Series of Subplot (c) at $\mathrm{T}=127,159,199$ (from Top to Bottom). (e) and (f) Show Differential Localization of Different Frequencies 


\section{S Transform based on Energy Normalized Window and the Corresponding TT Transform}

The analyzing time width of windows will be decreased adaptively with the increasing of frequency in standard S transform, which lead to different time-frequency resolution in timefrequency plane. But the windows of $S$ transform aren't energy normalized and timefrequency spectrum will generate obviously weighted effect with the increasing of frequency, which can't describe the distribution characteristic of spectrum in time-frequency plane and restrict analyzing ability of S transform. So, an energy normalized window whose width is related to frequency is defined:

$$
w_{N}(t)=\frac{|\delta f|^{\frac{1}{2}}}{\pi^{\frac{1}{4}}} \exp \left(-(\delta f t)^{2} / 2\right)
$$

In (9), the width of $W_{N}(t)$ is inversely proportional to $f$, and $\delta$ adjusts the change speed. The window function $W_{N}(t)$ satisfies the energy normalized condition:

$$
\int_{-\infty}^{+\infty}\left|w_{N}(t)\right|^{2} d t=1
$$

Therefore, $\mathrm{S}$ transform based on (9) is defined as:

$$
S_{N}(\tau, f)=\int_{-\infty}^{+\infty} u(t) \frac{|\delta f|^{\frac{1}{2}}}{\pi^{\frac{1}{4}}} \exp \left(-(\delta f(t-\tau))^{2} / 2\right) \exp (-i 2 \pi f t) d t
$$

Where $\tau$ is the center of $W_{N}(t)$ and controls the time axis position of the window, $\delta$ adjusts the change speed of window width with frequency to adapt different timefrequency resolution. Energy normalized $S$ transform has the advantage of standard $S$ transform such as the width of window adaptively changing with frequency and not need to meet wavelet admissibility condition. In addition, it has the same computation efficiency as standard S transform because only the coefficients of the widows are different and both of the two transform can be computed by using FFT (Fast Fourier Transform). According to inverse Fourier transform, original signal can be reconstructed perfectly from $S_{N}(\tau, f)$ :

$$
U(f)=\frac{\sqrt{|\delta f| / 2}}{\pi^{1 / 4}} \int_{-\infty}^{+\infty} S_{N}(\tau, f) d \tau
$$

Similarly, the discrete form of (11) is

$$
S_{N}(j T, n / N T)=\frac{1}{N} \sum_{m=0}^{N+1} U\left[\frac{m+n}{N T}\right] \pi^{0.25} \sqrt{\frac{2 N T}{\delta n}} \exp \left(-\frac{2 \pi^{2} m^{2}}{\delta^{2} n^{2}}\right) \exp \left(i \frac{2 \pi m j}{N}\right)
$$

Where $T$ is sampling interval, $N$ is the length of time series. Figure 2(a) is a synthetic time series, (b) is standard S transform of (a), (c) and (d) are energy normalized S transform of (a) corresponding to $\delta=1$ and $\delta=0.5$ respectively. It can be seen that the time and frequency resolution of both the transform is same ( $\delta=1$ for energy normalized $S$ transform), but the characteristic of energy distribution of different frequency contents is distrinct, especially for high frequency contents, the energy intensity is small obviously. Figure 2(d) shows the spectrum corresponding to $\delta=0.5$, compared with Figure 2(c), its frequency resolution is higher, which illustrates that $\delta$ can change time-frequency resolution. 


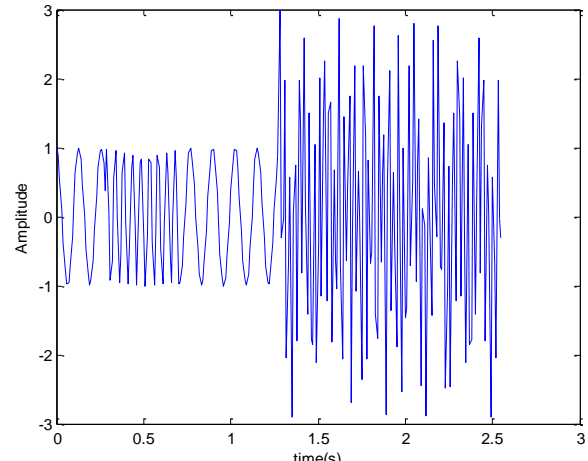

(a)

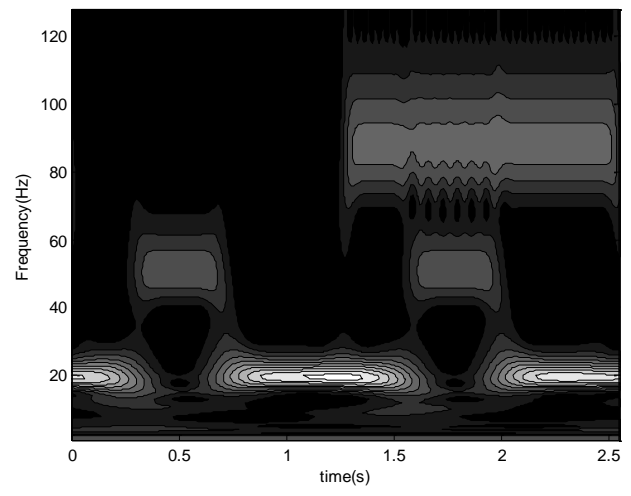

(c)

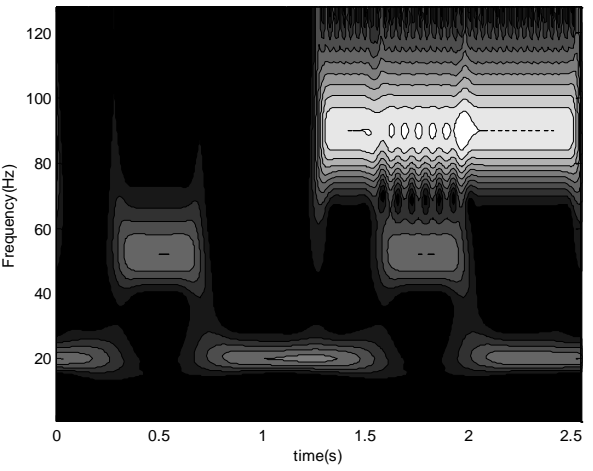

(b)

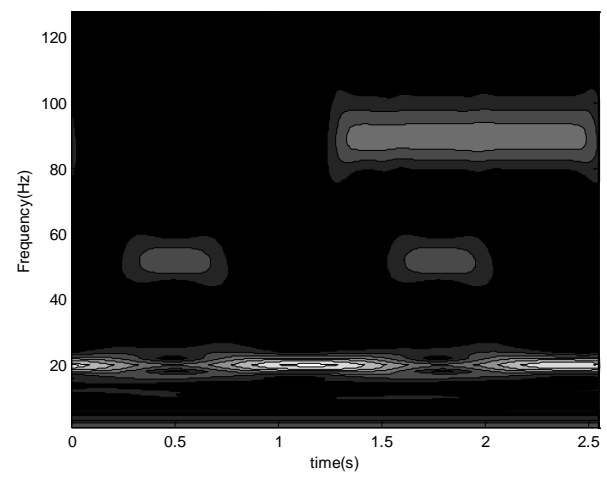

(d)

Figure 2. (a) Testing Signal. (b) Standard S Transform of (a). (c)-(d) Energy Normalized S Transform Corresponding to $\delta=1$ and $\mathbf{0 . 5}$

In order to embody time-frequency concentration of energy normalized $\mathrm{S}$ transform for non-stationary signal, LFM (Linear Frequency Modulated) signal is used to compare. Figure 3(a) is a LFM signal, (b) is standard S transform of (a), (c) and (d) are energy normalized S transform of (a) corresponding to $\delta=1$ and $\delta=0.4$ respectively. Figure 3(b) and (c) have the same time-frequency concentration, but with increasing of frequency, the spectrum energy of (c) is lower obviously than (b), which reduces the weight effect of window in standard $S$ transform. (d) Also shows the influence of $\delta$ to the time-frequency resolution. (e) is the frequency spectrum when $t=1.5 \mathrm{~s}$, where the amplitude of high frequency contents is smaller than that of energy normalized $\mathrm{S}$ transform.

It can be seen from Figure 3 that normalized $S$ transform has more precisely high frequency resolution, which means that the transform can extract high frequency effectively. Similar to discussion in section 2, we also can obtain TT transform corresponding to energy normalized S transform. Figure 4(a) is the normalized S transform and TT transform of Figure 1(a). It can be seen that the spectrum density of high frequency component is smaller than that of standard S transform obviously. From time index 0 to 127 , the low frequency component is also prominent, although the higher frequency content is added from time index 30 to 70, but it is restrained, which also can be seen from Figure 4(b) such as $\tau=19$ and $\tau=49$. The same result also can be seen in Figure 5, where the signal is the same as figure 3(a). 


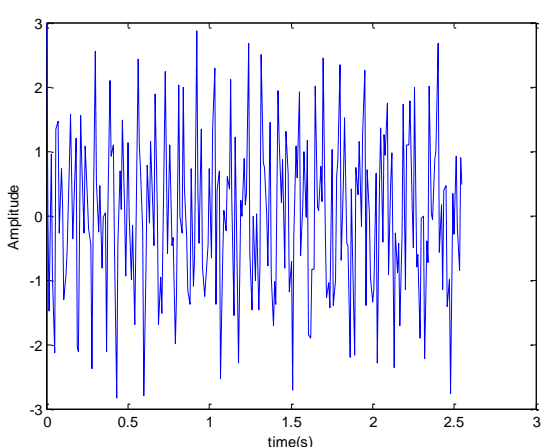

(a)

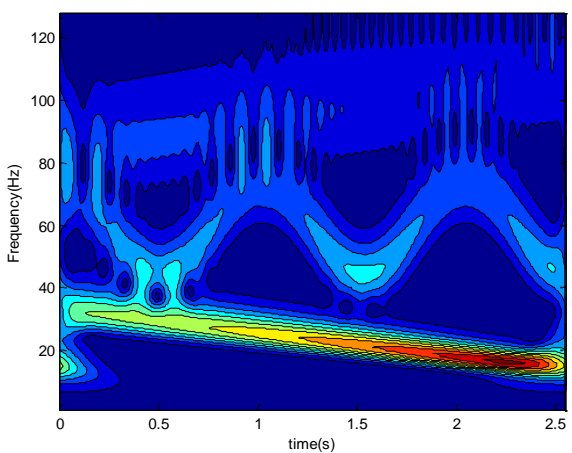

(c)

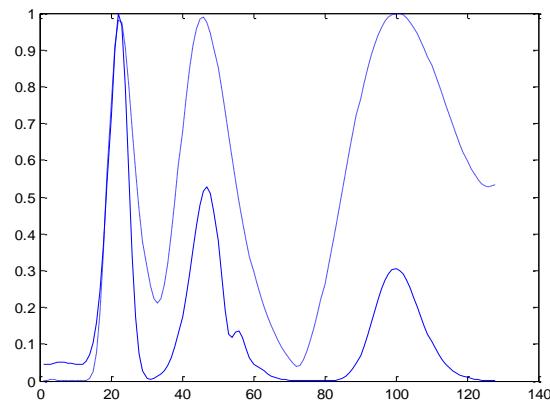

(e)

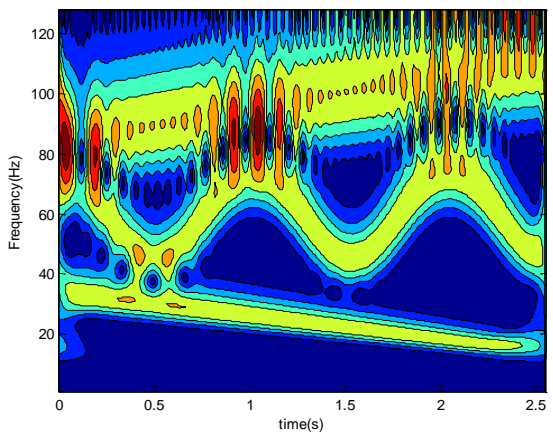

(b)

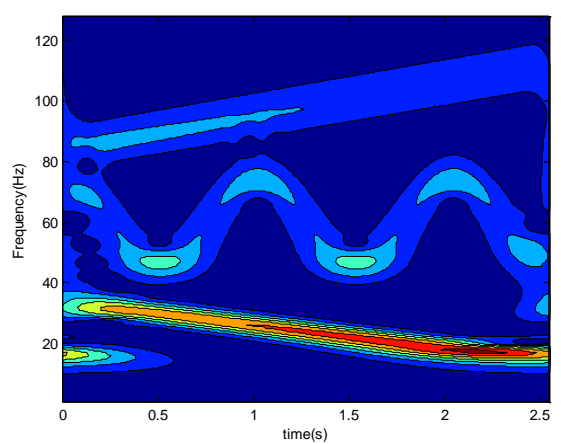

(d)

Figure 3. (a) LFM Signal. (b) Standard S Transform of (a). (c)-(d) Energy Normalized $\mathbf{S}$ Transform $(\delta=1$ and $\delta=0.4$ ). (e) The Frequency Spectrum when $\mathrm{t}=1.5 \mathrm{~s}$.

\section{Time-Frequency and Time-Time Filtering}

TT transform can concentrate high frequency content on its diagonal and low frequency components can be filtered by extracting the elements near the diagonal. But part of the high interference also is survived and some effective low frequency content is left out during filtering. The scheme that time-frequency filter based on energy normalized $\mathrm{S}$ transform is used to eliminate high frequency interference first and TT transform is adopted to filter low frequency interference is introduced here. 
According to energy normalized $\mathrm{S}$ transform, the time-frequency filter can be designed as follows:
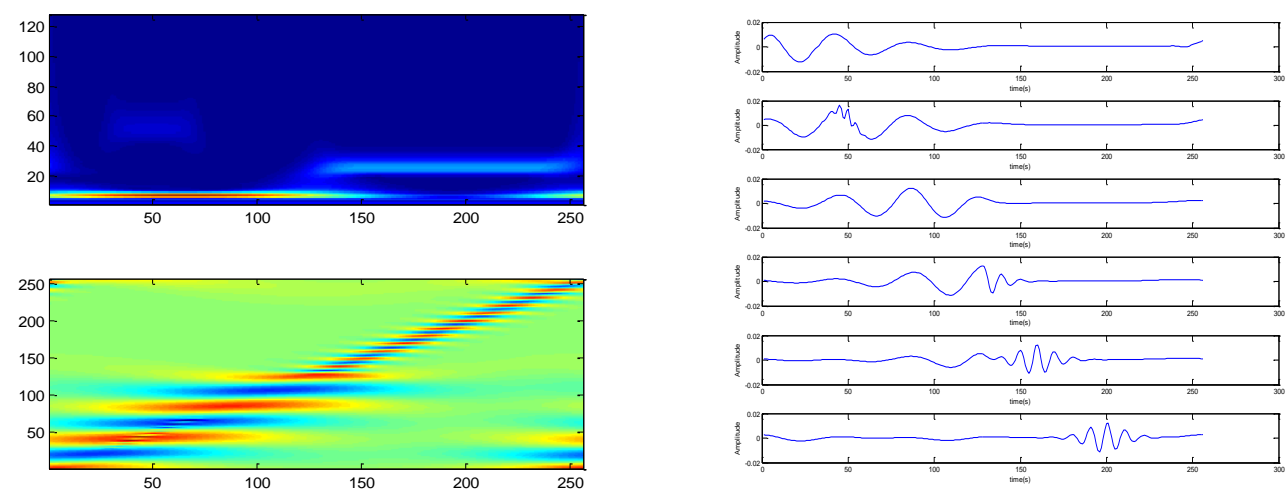

(a)

(b)

Figure 4. (a) Normalized S Transform and TT Transform of Figure 1(a). (b) TTSeries of Subplot (a) at $\mathrm{T}=19,49,89,127,159,199$

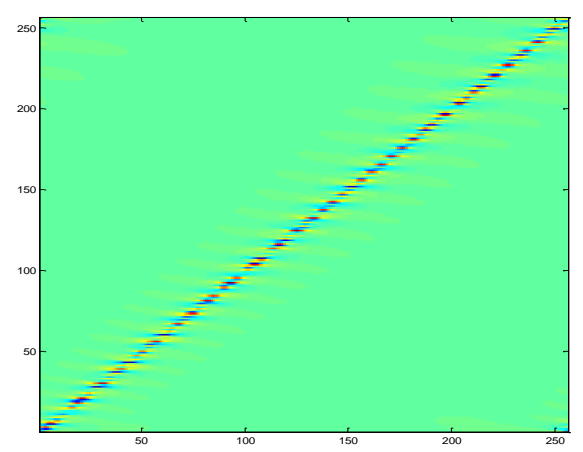

(a)

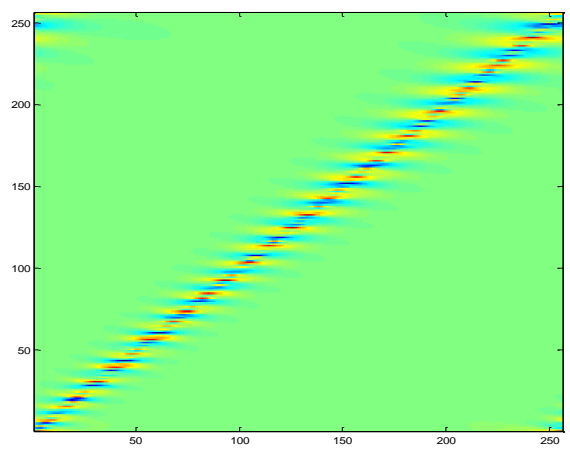

(b)

Figure 5. (a) TT Transform of Standard S Transform. (b) TT Transform of Normalized S Transform

$$
\int_{-\infty}^{+\infty} S_{N}(\tau, f) d \tau=\int_{-\infty}^{+\infty} \int_{-\infty}^{+\infty} u(t) \frac{|\delta f|^{\frac{1}{2}}}{\pi^{\frac{1}{4}}} \exp \left(-(\delta f(t-\tau))^{2} / 2\right) \exp (-i 2 \pi f t) d t d \tau
$$

Let $\beta=t-\tau$, then:

$$
\begin{gathered}
\int \frac{|\delta f|^{1 / 2}}{\pi^{1 / 4}} \exp \left(-(\delta f \beta)^{2} / 2\right) d \beta=\pi^{1 / 4} \sqrt{2 /|\delta f|} \\
\int_{-\infty}^{+\infty} S_{N}(\tau, f) d \tau=\int_{-\infty}^{+\infty} u(t) \pi^{\frac{1}{4}} \sqrt{2 /|\delta f|} \exp (-i 2 \pi f t) d t=\pi^{\frac{1}{4}} \sqrt{2 /\left.\delta f\right|^{U}}(f)
\end{gathered}
$$

Therefore:

$$
U(f)=\pi^{1 / 4} \sqrt{|\delta f| / 2} \int_{-\infty}^{+\infty} S_{N}(\tau, f) d \tau
$$


The time-frequency filter is:

$$
\begin{array}{r}
u_{f}(t)=\int_{-\infty}^{+\infty} \pi \sqrt[1]{4} \sqrt{|\delta f| / 2} \int_{-\infty}^{+\infty} S_{N}(\tau, f) H(\tau, f) d \tau \exp (i 2 \pi f t) d f \\
H(\tau, f)=\left\{\begin{array}{lc}
1 & f \in\left[\frac{f_{i}-f_{B}}{2}, \frac{f_{i}+f_{B}}{2}\right] \\
0 & \text { others }
\end{array}\right.
\end{array}
$$

Where $H(\tau, f)$ is time-frequency filter, $f_{i}$ is instantaneous frequency, $f_{B}$ is bandwidth of filter, which is defined according to the energy concentration measurement along instantaneous frequency in time-frequency plane.

Figure 6(a) is a seismic record which is a classic non-stationary signal; (b) is its time-frequency spectrum. It can be seen that the interference includes high interference, random noise and low frequency surface wave. (d) is the time-frequency spectrum after filtered by (23), where high interference and random noise are eliminated effectively. (e) is the TT transform of filtered energy normalized $S$ transform, compared to the TT transform of original signal, the high frequency contents is restrained obviously. (f) is the energy normalized S transform after TT transform filtering, compared with (d), the low frequency contents is filtered efficiently and the high effective content is preserved. (g) is the reconstructed signal where interference is restrained efficiently reflected wave is highlighted. (h) Is the error between the original signal and the reconstructed signal.

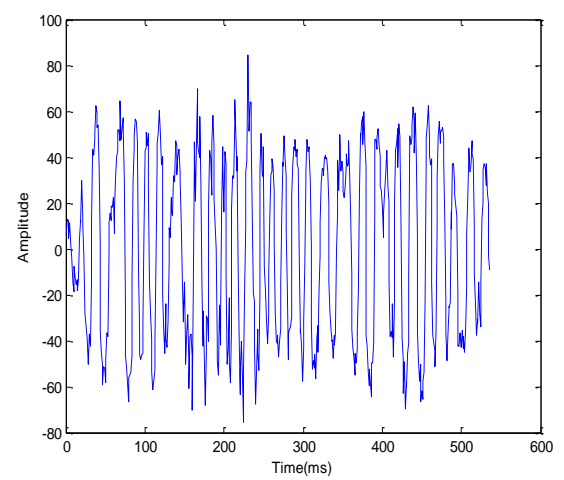

(a)

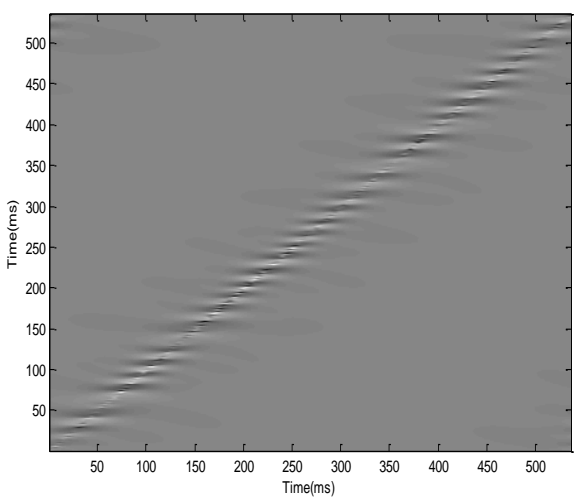

(c)

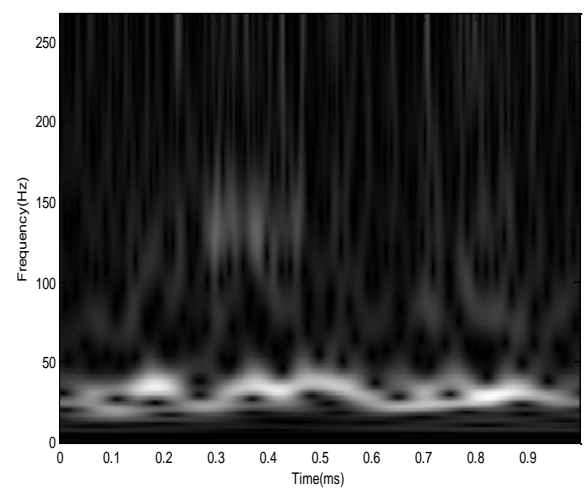

(b)

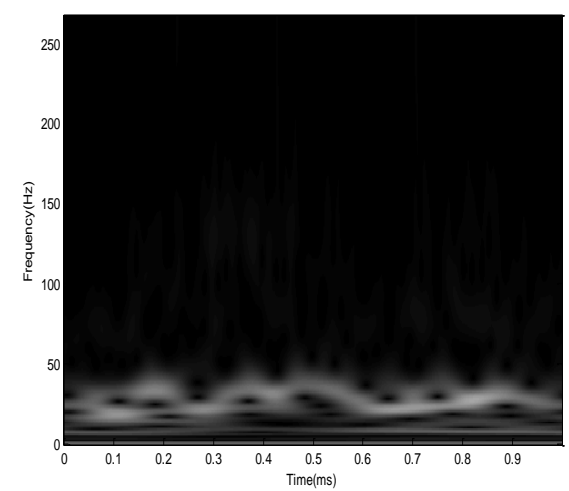

(d) 


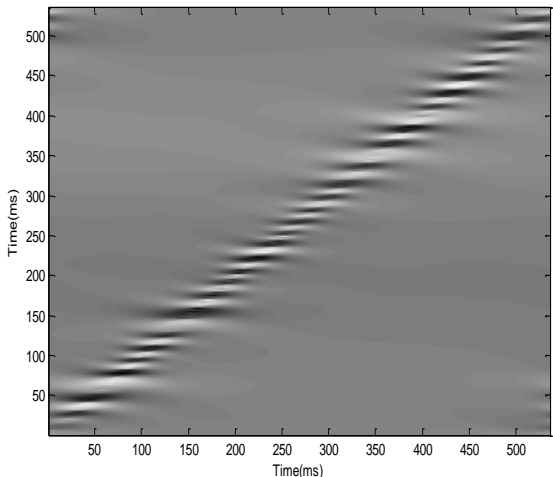

(e)

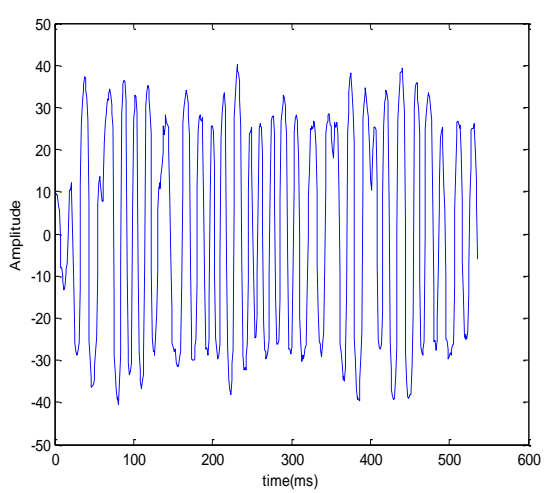

( $g$ )

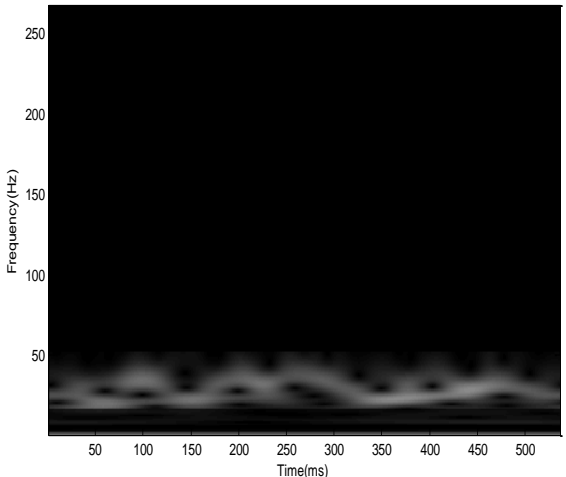

(f)

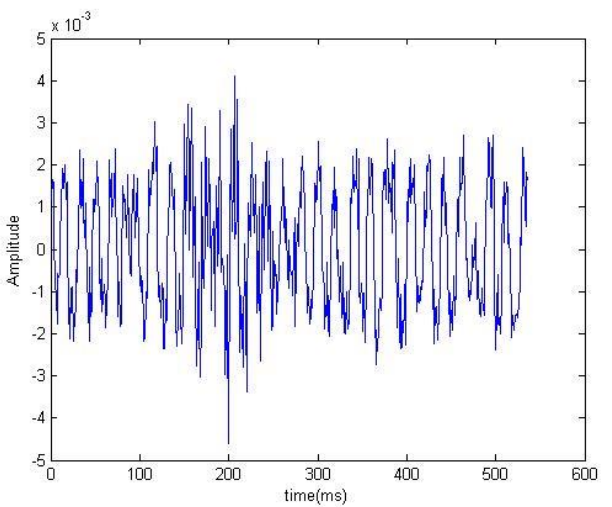

(h)

Figure 6. (a) Seismic Signal. (b) Time-Frequency Spectrum of (a). (c) TT Transform of (b). (d) Time-Frequency Filtering Spectrum. (e) TT Transform of (c). (f) Energy Normalized S Transform. (f) Spectrum After TT Filtering. (g) Reconstructed Signal. (h) Error Signal.

The filtering combined time-frequency and time-time filter based on energy normalized $\mathrm{S}$ transform and TT transform respectively is used to seismic profile. The method is described as follows:

(1) Every trace seismic record is filtered by the time-frequency filter according to energy normalized S transform, which the high frequency interference and random noise are restrained.

(2) The TT transform of records after suppressed high frequency and random noise is obtained and the diagonal elements are extracted, which the low frequency interference such as surface wave etc., is restrained.

Figure 7 is the processed results, where Figure 7(a) is the original profile and (b) is the filtered profile and (c) is the error profile. The high and low frequency interference is suppressed efficiently and the reflected events become more continuous, which improves the resolution of seismic records. 


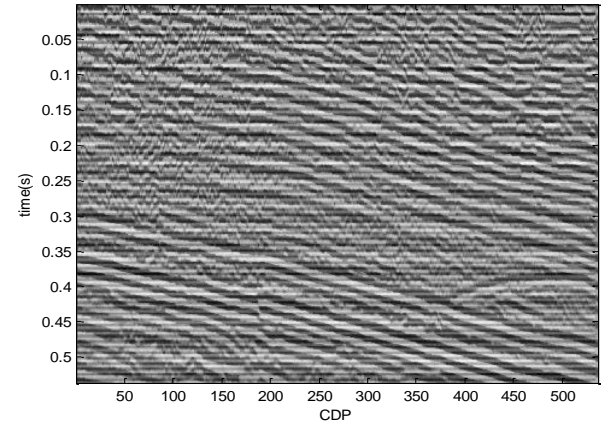

(a)

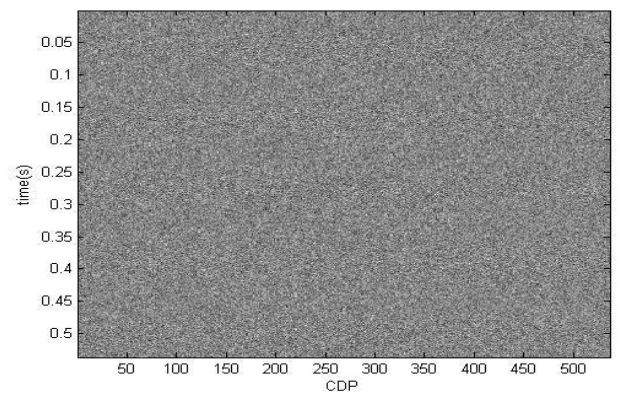

(c)

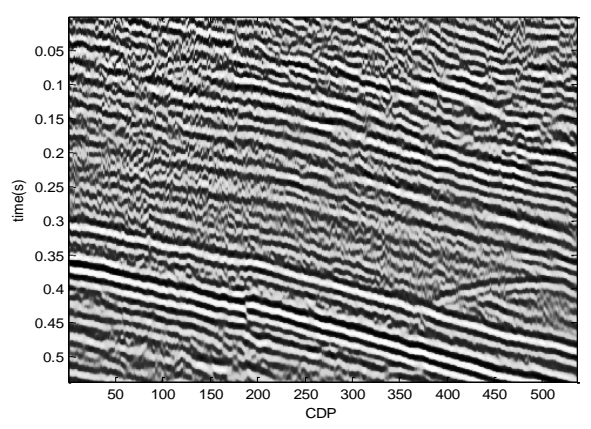

(b)

\section{Figure 7. (a) Original Seismic Profile. (b) Filtered Seismic Profile. (c) Error Profile}

\section{Conclusions}

The time-frequency filtering approach based on energy normalized $\mathrm{S}$ transform and TT transform is discussed, where the $\mathrm{S}$ transform alleviate the weighted influence of time-frequency spectrum. High frequency clutters are restrained by the $S$ transform and TT transform filters low frequency interference. The combined filtering can overcome the defect of TT transform which has obvious suppression for low frequency, but it also reserves part high frequency interference and leave out some low frequency useful contents. The feasibility of the method is verified through filtering seismic signal which can avoid some pseeudomorph.

\section{Acknowledgements}

The work is sponsored by Education Department of Heilongjiang province (NO.12531063) and Chang Jiang Scholar Candidates Programme for Provincial Universities in Heilongjiang (2012CJHB005).

\section{References}

[1] L. Cohen, "Time-frequency analysis: Theory and application", Englewood Cliffs, NJ: Prentice Hall, Inc. (1995).

[2] S. G. Mallat, "A Wavelet Tour of Signal Processing”, Academic Press, San Diego, Calif, USA, 2nd edition, (1999).

[3] R. G. Stockwell, "S-Transform Analysis of Gravity Wave Activity from a Small Scale Network of Airglow Imagers", The University of Western Ontario, (1999), pp. 80-96. 
[4] S. Hasheminejad, S. Esmaeili and S. Jazebi, "Power quality disturbance classification using S-transform and hidden markov model”, Electric Power Components and Systems, no. 40, (2012), pp. 1160-1182.

[5] L. Xiuwen, Y. Jianhong, L. Min and X. Jinwu, "A time-frequency filtering method based on generalized S transform and its application in machinery fault diagnosis", Applied Mechanics and Materials, vol. 157-158, (2012), pp. 531-537.

[6] Z. Min, C. Wenjing and J. Mohua, "Application of S-transform profilemetry in eliminating nonlinearity in fringe pattern", Applied Optics, no. 51, (2012), pp. 577-587.

[7] L. Jicheng, M. Dapeng and L. Mengda, "S-transform and its application in the spectrum analysis of seismic signal", Lecture Notes in Electrical Engineering, no. 139, (2012), pp. 81-86.

[8] J. Liu, J. Yao and X. Liu, "Generalized S Transform with Adaptive Optimized Window and Application in Seismic Signal Analysis”, Information Technology Journal, no. 12, (2013), pp. 276-286.

[9] N. Z. Mohamad and A. F. Abidin, "S-Transform based technique to detect high resistance symmetrical fault during power swing", IEEE 8th International Colloquium on Signal Processing and Its Applications, (2012) March 23-25, pp. 282-286, Melaka, Malaysia.

[10] C. R. Pinnegar and L. Mansinha, "A method of time-time analysis:The TT-transform”, Digital Signal Process, no. $13,(\mathbf{2 0 0 3})$, pp. 588-603.

[11] C. R. Pinnegar, "Time-frequency and time-time filtering with the S-transform and TT-transform", Digital Signal Process, no. 15, (2005), pp. 604-620.

[12] F. Xian-feng and Z. Ming, "Gearbox fault detection using Hilbert and TT-transform", Key Engineering Materials, no. 7, (2005), pp. 79-86.

[13] C. R. Pinnegar, "Generalizing the TT-transform”, Digital Signal Processing, no. 19, (2009), pp. 144-152.

[14] S. C. M. Schimmel and J. Danobeitia, "On the TT-transform and its diagonal elements", IEEE Trans on Signal Processing, no. 11, (2008), pp. 5709-5714.

[15] C. R. Pinnegar and L. Mansinha, "The S-transform with windows of varying and arbitrary shape", Geophysics, no. 68, (2003), pp. 381-385.

[16] C. R. Pinnegar and L. Mansinha, "The bi-Gaussian S-transform”, SIAM J.Sci.Comput., no. 24,(2003), pp. 1678-1692.

\section{Authors}

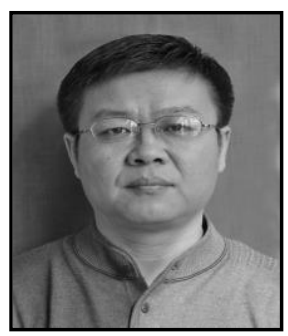

Jicheng Liu, he is a full professor in the school of computer in School of Electrical and Information Engineering, University of Northeast Petroleum, China. He obtained his $\mathrm{PhD}$ degree from Beihang University (BUAA), China, 2003. His research interests mainly include non-stationary signal processing and seismic signal processing.

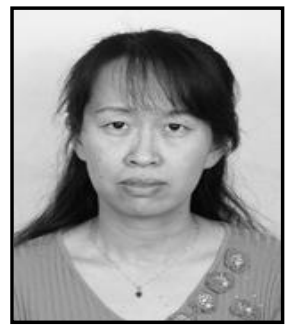

Jianhong Yao, she is a full professor in School of Electrical and Information Engineering, University of Northeast Petroleum, China. She obtained her $\mathrm{PhD}$ degree from University of Northeast Petroleum in 2010. Her research interests are intelligent signal processing.

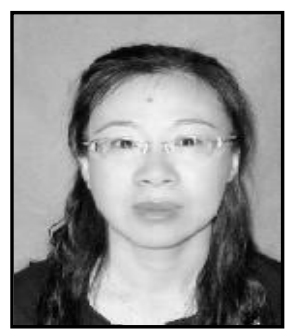

Xia Liu, she is a full professor in School of Electrical and Information Engineering, University of Northeast Petroleum, China. She obtained her Master degree from University of Northeast Petroleum in 2001. Her research interests are signal detection technology. 